\title{
NOTAS HISTÓRICAS SOBRE A FAMÍLIA CACTACEAE NO RIO GRANDE DO SUL (BRASIL) E URUGUAI. III - PERÍODO CONTEMPORÂNEO (1980-2017): TAXONOMISTAS GAÚCHOS, URUGUAIOS E EUROPEUS ${ }^{1}$
}

\author{
RODRIGO CORRÊA PONTES ${ }^{2}$ JOSÉ NEWTON CARDOSO MARCHIORI ${ }^{3}$ \\ LEOPOLDO WITECK NETO ${ }^{4}$
}

\section{RESUMO}

É apresentada a terceira e última parte da história das descobertas pertinentes à família Cactaceae no Rio Grande do Sul e Uruguai.

Palavras-chave: Botânica, Cactaceae, História, Rio Grande do Sul, Uruguai.

\section{ABSTRACT}

[Historical notes about the Cactaceae family in the State of Rio Grande do Sul (Brazil) and Uruguay. III - Contemporary period (1980-2017): Plant taxonomists from Rio Grande do Sul State, Uruguay and Europe].

The third and last period of the History about Cactaceae findings in Rio Grande do Sul and Uruguay is presented.

Key words: Botany, Cactaceae, History, Rio Grande do Sul, Uruguay.

\section{INTRODUÇÃO}

Com o descobrimento de novidades taxonômicas no Rio Grande do Sul e Uruguai durante a década de setenta do século vinte, muitos colecionadores e entusiastas europeus vieram à América do Sul em busca de novas espécies. Cabe ressaltar que a atividade exploratória nesse período foi majoritariamente desenvolvida por pessoas com especial interesse na formação de coleções de plantas ornamentais, mas que, amadurecendo suas perspectivas, passaram a colaborar com renomados botânicos no repasse de informações, marcando um novo período na história dos cactos.

1 Recebido em 28-5-2017 e aceito para publicação em 21-6-2017.

2 Geógrafo, doutorando em Geografia, Programa de PósGraduação em Geografia, Universidade Federal de Santa Maria.rodrigocorreapontes@gmail.com

3 Engenheiro Florestal, Dr. Bolsista de Produtividade em Pesquisa (CNPq-Brasil). Professor Titular do Departamento de Ciências Florestais, Universidade Federal de Santa Maria.marchiori@pq.cnpq.br

4 Engenheiro Florestal, M.e. Professor do Colégio Politécnico, Universidade Federal de Santa Maria. lwiteck@gmail.com
Neste período, é igualmente nítido o desenvolvimento de estudos botânicos a partir de universidades, com resultados muito importantes para o conhecimento das espécies e distribuição das mesmas.

No início da década de 1980, novos excursionistas europeus estiveram na América do Sul em busca de cactos. É o caso, entre outros, de Heinz Werner Ruoff-Vetsch (02-04-1946 - 0302-1994), nascido em Küssnacht am Rigi, Suíça (Gerloff, 1994a). Sua paixão pelos cactos começou em 1968, ao presentear a esposa com uma dessas plantas no dia das mães. $\mathrm{Na}$ sequência, ele construiu uma pequena estufa para plantas e se especializou no cultivo de cactos uruguaios. Em 14-04-1982, juntamente com Peter Schlosser, ele encontrou no norte do Uruguai dois exemplares de um novo táxon, descrito, posteriormente, como Notocactus ruoffii (Ruoff, 1989). Ele também contribuiu com artigos na revista "Internoto", especializada no gênero Notocactus.

Na primeira metade dos anos oitenta, destacaram-se os cactólogos a seguir comentados na busca de cactos pelo Rio Grande do Sul e Uruguai. 
Entusiasta e autodidata, Karl Heinz Prestlé (21-06-1925 - 13-05-2016) nasceu em Wuppertal, Alemanha (Prauser, 2016) e, na Segunda Guerra Mundial, integrou a Marinha de Guerra alemã (Kriegsmarine). Mais tarde, atuou como analista químico em uma grande empresa de tintas da Europa e, na década de cinquenta, por motivos profissionais, transferiu-se com a família para Veghel, na Holanda. Sua paixão pelos cactos surgiu de forma inusitada e mediante sua esposa, que lhe presenteou com um cacto recebido de sua dentista. Ao longo do tempo, Prestlé foi aperfeiçoando as técnicas de cultivo nas estufas de sua casa e a "febre" pelos cactos levou-o a querer visitar e estudar os mesmos nos seus habitats naturais. Em sua primeira viagem à América do Sul, feita em 1976, ele percorreu diversas localidades do Uruguai com Hugo Schlosser, encontrando, como novidades, novas formas botânicas e variedades de Gymnocalycium, Frailea e Notocactus.

De 1979 a 1985, Prestlé viajou com Francisco (Chico) Stockinger, Ari Nilson e Antônio Gutierrez (Prestlé 1980, 1981a, 1981b). Na década de oitenta, suas explorações no Rio Grande do Sul renderam novidades, por ele descritas como Notocactus ibicuiensis, $N$. stockingeri, $N$. macambarensis, N. meonacanthus e $N$. gibberulus (Prestlé 1985a, 1985b, 1986a, 1986b, 1986c).

Devido a atritos com Francisco Stockinger, motivados, talvez, pela primazia dos novos achados, Prestlé viajou a partir de 1986 com José Alberto Feeburg (27-12-1957), natural de Porto Alegre.

Prestlé descreveu e introduziu em cultivo muitos ecótipos e exemplares selecionados de populações, os quais, em sua concepção, constituíam novas espécies. A maioria de suas descrições taxonômicas não teve diagnose em latim. Devido à criação desnecessária e/ou indevida de novos táxons, o trabalho de Prestlé perdeu muito em credibilidade, por mais agregar problemas taxonômicos à família Cactaceae do que contribuir, efetivamente, à mesma.
Prestlé publicou diversos artigos em revistas especializadas, salientando-se um trabalho dedicado ao gênero Gymnocalyclium no Rio Grande do Sul (Prestlé, 1991).

Sua obra mais expressiva - "Die Gattung Frailea" -, aborda o gênero com visão muito pessoal, configurando uma desastrosa tentativa de vincular a distribuição e evolução de suas espécies com a geologia. Além desse aspecto, Prestlé estabeleceu oitenta novos táxons, aproximadamente, muitos deles sem holótipo definido e diagnose em latim, além de variedades e formas botânicas, com base em coletas próprias e de outros exploradores do Rio Grande do Sul e Uruguai (Prestlé, 1998).

Outro notável colecionador de cactos foi Francisco Alexandre Stockinger (07-08-1919 12-04-2009), natural de Traun, Áustria. Em 1921, ele e sua família imigraram para o Brasil, estabelecendo-se no município de Santo Anastácio, interior de São Paulo. Em 1939, formou-se em Meteorologia e, sete anos mais tarde, iniciou estudos artísticos no Liceu de Artes e Ofícios do Rio de Janeiro (Alves, 2012). Em 1952, Stockinger mudou-se para Porto Alegre e, dois anos mais tarde, naturalizou-se brasileiro. Sua produção artística abrange a elaboração de charges, publicadas em jornais, obras em xilogravura e, principalmente, esculturas em mármore e bronze. Stockinger participou de diversas exposições nacionais e internacionais de arte, notabilizando-se como um dos mais importantes escultores brasileiros da segunda metade do século vinte.

A relação de Stockinger com os cactos surgiu em período muito difícil de sua vida, por questão de saúde. Em 1974, após cirurgia cardíaca, ele contraiu uma grave hepatite pós-operatória, que o levou a ficar em repouso por quase um ano (Alves, 2012). Na busca de ocupação para o tempo livre, Ieda, sua esposa, presenteou-lhe com um livro sobre cactos, motivando "Xico", como era conhecido pelos amigos, a dedicar-se ao cultivo e estudo dessas plantas. 
De acordo com suas anotações, Stockinger realizou muitas viagens ao final dos anos setenta, mas sem registrar dados. A partir de 1979, o escultor viajou com Ari Delmo Nilson (25-071955), técnico agrícola natural de Marcelino Ramos e funcionário do Jardim Botânico de Porto Alegre. Ao encontrar parte da coleção de cactos iniciada por Carlos Zuckermann na década de sessenta, Nilson decidiu reorganizá-la ao final dos anos setenta, estabelecendo uma forte ligação com o conhecimento dessa família botânica (Nilson, comunicação pessoal, 2016). Com olhar atento aos cactos, as viagens de campo de Nilson e Stockinger, promovidas, financeiramente, pelo último, na maioria das vezes, levou os parceiros a conhecer boa parte do interior do Rio Grande do Sul e Uruguai, ampliando o conhecimento de ambos sobre a distribuição das espécies. O êxito dessas viagens atraiu a atenção de grandes especialistas europeus, como Karl Heinz Prestlé, Wolf-Rainer Abraham e Norbert Gerloff, que também passaram a excursionar pelo Rio Grande do Sul e Uruguai. Na segunda metade dos anos noventa, Stockinger decidiu focar sua atenção no gênero Frailea, do qual se tornou autoridade reconhecida. Com o avanço da idade e problemas de saúde, Stockinger reduziu as saídas a campo, realizando viagens mais curtas e em períodos de temperatura não tão elevadas.

Outro companheiro de Stockinger e Nilson, em suas viagens, foi o artista plástico e pintor Antonio Carlos Gutierrez Assumpção (11-101934 - 31-12-2004). Nascido e criado em Maçambará (na época, uma simples localidade do município de Itaqui), Gutierrez transferiu-se aos 23 anos para Porto Alegre, onde foi aluno de Iberê Camargo, um dos mais famosos artistas plásticos gaúchos, retornando, mais tarde, a seu recanto em Maçambará, onde viveu pelo resto da vida (Duarte, 1992). Apesar de seu estilo ermitão, ele é reconhecido como um dos mais importantes artistas plásticos gaúchos da segunda metade do século vinte e como um dos introdutores da arte moderna no Rio Grande do Sul. Sua arte, inspirada em paisagens e costu- mes gaúchos, situa-se entre o surrealismo e o metafísico (Duarte, 1992). Além de manter uma bela coleção de cactos, Gutierrez também foi um ecologista militante no oeste do Estado, tendo atuado, inclusive, como fiscal do Ibama.

Em suas viagens a estâncias na região da campanha, Gutierrez realizava coletas e registros dos cactos encontrados. Encarregado de tomar conta de uma fazenda em Uruguaiana, ele veio a encontrar o belíssimo Notocactus gutierrezii, espécie endêmica e sabidamente rara no Rio Grande do Sul. Infelizmente, muito pouco do material original do coletor permanece em cultivo na Europa. Após sucessivas cirurgias para o tratamento de um câncer, no qual, inclusive, esteve por três meses em coma, acabou falecendo aos 70 anos de idade. Como homenagem póstuma, Gutierrez empresta seu nome à biblioteca pública municipal de Maçambará, como forma de reconhecimento por seu pioneirismo.

O taxonomista mais ativo na região, na década de oitenta, foi, sem dúvidas, o Dr. WolfRainer Abraham (26-04-1952), biólogo e botânico alemão que deixou importantes contribuições para o Rio Grande do Sul e Uruguai. Consta que seu interesse por cactos teve início ao receber um pequeno exemplar de sua esposa, como presente. Em 1982, ele realizou sua primeira viagem à América do Sul, percorrendo áreas no Rio Grande do Sul e Uruguai em companhia de Hugo Schlosser, com quem adquiriu um vasto conhecimento na identificação de cactos.

Três anos mais tarde, Abraham viajou com Antonio Gutierrez, Ari Nilson e Francisco Stockinger pelas regiões sul e oeste do estado gaúcho, bem como em parte do Uruguai, em busca de novas espécies e populações. Em 1987, com Rudi Büneker, a excursão, de quatro meses, abrangeu grande parte do estado do Rio Grande do Sul.

Além de seus próprios achados, Abraham descreveu novidades de Schlosser, Stockinger e, principalmente, de Büneker, tais como: Notocactus scopa var. xiphacanthus, N. scopa var. machadoensis, N. scopa var. xicoi, N. 
rudibuenekeri, Wigginsia schaeferiana, Notocactus rubrigematus, N. gutierrezii, N. scopa var.marchesii, Frailea buenekeri, Notocactus linkii var. albispinus, Frailea buenekeri e Notocactus megapotamicus var. multicolorispinus (Abraham, 1987, 1988a, 1988b, 1988c, 1988d, 1988e, 1988f, 1989, 1990a; 1990b; Abraham et Büneker, 1992). Abraham ainda prossegue com seus estudos sobre cactos do Rio Grande do Sul e Uruguai, publicando, periodicamente, artigos na revista Internoto, entre outras.

Dentre os novos táxons, salientam-se as descrições de Notocactus arnostianus e $N$. ritterianus pelos entusiastas Jiri Kolarik (20-021957) e Kamil Lisal (07-02-1952), feitas a partir de plantas importadas pelo viveiro Uhlig e procedentes de coletas realizadas por Horst \& Uebelmann no norte de Dom Pedrito (Lisal \& Kolarik, 1986).

Mesmo não tendo conhecido o Rio Grande no Sul e Uruguai, o renomado taxonomista alemão Pierre Joseph Braun (22-04-1959), de Köln (Colônia, Alemanha), descreveu uma nova variedade para o Rio Grande do Sul - Notocactus graessneri var. microdasys, (Braun, 1986) -, assim como elaborou novas combinações para os gêneros Frailea, Gymnocalycium e, sobretudo, transferiu espécies de Notocactus para Parodia (Hofacker et Braun, 1998; Braun et Esteves, 2001; Hunt 2006).

Após o falecimento de Leopoldo Horst, em 1987, emergiu um novo e importante protagonista na história sul-rio-grandense dos cactos. Ocorre que Werner Uebelmann prosseguiu em suas explorações e, hospedado no Polartur Hotel, de Estrela (RS), veio a conhecer Marino Gustavo Hamester (30-06-1937), natural da mesma cidade, que aceitou o convite para ser seu intérprete e guia nas viagens pelo Rio Grande do Sul e outros estados do Brasil (Hamester, comunicação pessoal, 2016).

Uebelmann e Hamester, acompanhados de Konrad Herm (08-08-1951), de Völkersbach (Alemanha), viajaram pelo Rio Grande do Sul e Uruguai de 01-10-1988 a 25-10-1988, prosse- guindo, após essa data, pelo sudeste e nordeste do Brasil. Posteriormente, Herm publicou, em revistas especializadas, artigos ricamente ilustrados sobre as espécies encontradas, bem como algumas revisões taxonômicas sobre o complexo Notocactus horstii, salientando-se a descrição de N. horstii fa. muglianus (Herm, 1993). Após essa longa excursão de quatro meses, aproximadamente, a dupla deixou de viajar em virtude de atritos surgidos pelo descumprimento de acordos da parte de Uebelmann em relação à Hamester.

Em 1989, Hamester viajou com Andreas Hofacker (04-04-1960), natural de Stuttgart (Alemanha), reencontrando uma variedade há muitos anos perdida - Notocactus arechavaletae var. horstii (Hofacker, 1990) -, e, como novidade, Frailea buenekeri subsp. densispina (Hofacker \& Herm, 1997). Nos anos de 1990, 1992 e 1994, Hofacker viajou com Kurt Ingo Horst e, mais tarde, sozinho.

Em parceria com Herm, Hofacker descreveu Notocactus minusculus e sua subspécie gravior (Hofacker \& Herm, 1996). Anos mais tarde, Hofacker, Herm e outros autores publicaram o livro Kakteen in Brasilien, com um capítulo inteiramente dedicado a espécies do Rio Grande do Sul (Herm et al., 2000). Em 2013, ele editou o livreto Notocactus, von Acanthocephala bis Wigginsia, ricamente ilustrado (Hofacker, 2013). Com frequência, Hofacker publica artigos sobre cactos do Rio Grande do Sul e Uruguai em periódicos europeus especializados, tais como Kakteen und andere Sukkulenten (Alemanha) e The Cactus Explorers (Inglaterra), prosseguindo, ainda, com suas viagens de investigação botânica.

Ainda em 1989, Marino guiou, em parte, a primeira viagem de Norbert Gerloff (15-081947) pela região. Natural de Ludwigsburg, Gerloff, embora diabético e portador de necessidades especiais, viajou vinte e duas vezes para a América do Sul entre os anos de 1989 e 2014, com o objetivo de estudar cactos pampianos da Argentina, Brasil (Rio Grande do Sul) e Uruguai. Como um dos fundadores e presidente, por 
muitos anos, da Internoto, a conhecida sociedade internacional dos amigos de Notocactus (fundada em 1980), Gerloff publicou, nesta revista, Notocactus scopa var. cobrensis, $N$. incomptus, $N$. glomeratus, $N$. uebelmannianus var. pleiocephalus, $N$. calvescens, $N$. langsdorffii fa. eddiewarasii, $N$. neohorstii var. prestlei $e$ Notocactus arachnites var. darilhoensis (Gerloff, 1990a, 1990b, 1991, 1994b, 1995, 1997; Gerloff et Könings, 1992; Gerloff et Nilson, 1994). No mesmo periódico, foram, também, publicados: Notocactus uebelmannianus var. nilsonii (Könings, 1990) e N. laetvirens fa. densispinus (Bergner, 1993).

Entre 1989-1994, Gerloff também realizou viagens com Ari Nilson, do Jardim Botânico de Porto Alegre. Ambos tiveram a idéia de elaborar uma coleção de cactos com ambientes representativos das regiões fitofisonômicas do Rio Grande do Sul, incluindo espécies endêmicas, inclusive de bromélias, plano que, infelizmente, acabou não realizado.

Gerloff ainda publicou o livro "Notokakteen - Gesamtdarstellung aller Notokakteen" e, nove anos mais tarde, uma revisão taxonômica, com novas combinações (Gerloff et al 1995; Gerloff et al., 2004). Em 2014, ele realizou sua última viagem e, por motivos de saúde, diminuiu a intensidade de seus estudos com cactos, doando grande parte de sua coleção em 2016 para Ernest Etzlstorfer, de Haag, Baixa Áustria.

Marino também realizou viagens com Wolfgang Gemmrich (10-12-1952, Erfurt, Alemanha), Jozka Neduchal (13-09-1932 - 24-102010, Pilsen, República Tcheca) e Stanislav Stuchlík (14-05-1943, Brno, República Tcheca) entre 1992 e 1998. Em 1993, Stuchlík publicou a interessante monografia "Rod Notocactus", inteiramente dedicada ao gênero Notocactus e editada na República Tcheca (Stuchlík, 1993).

Em 1998, Gemmrich trouxe uma amiga (Christa Koch) para conhecer as belezas do Rio Grande do Sul, oportunidade em que ambos encontraram Notocactus vilanovensis, em Vila Nova do Sul (Koch, 2003). Em 2002 e 2004,
Hamester novamente viajou com Stanislav Stuchlík e Wolfgang Gemmrich.

Stuchlík também promoveu viagens com outros europeus ao Rio Grande do Sul e Uruguai. De setembro a novembro de 2008, ele guiou Csaba Kádar (04.06.1962), natural de Baia Mare (Romênia), por diversos habitats no Rio Grande do Sul (Gerloff, 2010).

Em 2010, Stuchlík publicou um livreto com as espécies de cactos do Rio Grande do Sul (Stuchlík, 2010). Um ano mais tarde, ele percorreu áreas gaúchas com o polonês Tadeuz Nycz. Na primavera e verão de 2016, Stuchlík viajou com Peter Krämer, outro entusiasta de cactos e viajante alemão. Stuchlík é um dos idealizadores e redatores do periódico Minimus, revista tcheca fundada em 1991 e dedicada, exclusivamente, ao gênero Notocactus.

Como biólogo, João Felisberto Larocca e Silva (07-12-1965, Gravataí, RS) tem focalizado, em seus estudos com cactos, as curiosas estratégias de sobrevivência dos mesmos em afloramentos rochosos do Rio Grande do Sul. Em 1989, no início de sua carreira acadêmica, ele conheceu o ambientalista porto-alegrense José Antônio Kroeff Lutzenberger (17-12-1926 - 14-05-2002), que muito o incentivou à pesquisa e facilitou importantes contatos no mundo científico, entre os quais o botânico alemão Wilhelm Barthlott (22-06-1946, nascido em Forst, Baden), e o botânico suíço Urs Eggli (1102-1959, nascido em Zurique), possibilitando, dessa forma, a realização de estágios na Coleção Municipal de Suculentas (Zurique) e no Jardim Botânico de Hamburgo, instituições que reúnem destacadas coleções científicas de plantas suculentas (Larroca, comunicação pessoal, 2016). Sua dissertação de mestrado em Botânica (1994-1998), intitulada "Cactáceas em paredões rochosos da Serra Geral do Rio Grande do Sul: uma abordagem fitogeográfica", foi orientada pelo Dr. Jorge Luiz Waechter, professor da Universidade Federal do Rio Grande do Sul (Larroca, 1998). Também sob a orientação do Dr. Waechter, Danielle Bauer realizou uma 
importante contribuição ao conhecimento de cactos epifíticos do Rio Grande do Sul e sua distribuição geográfica (Bauer \& Waechter, 2006).

Outro pesquisador e estudioso de cactos do Rio Grande do Sul é Marlon Câmara Machado (05-05-1975, Cruz das Almas, Bahia), que realizou viagens com apoio de fundos doados por Werner Uebelmann para a pesquisa científica de cactáceas brasileiras e sua preservação. Machado ainda publicou interessantes artigos sobre o gênero Frailea no Rio Grande do Sul (2007a, 2007b), frutos de suas viagens realizadas em 2005, 2006 e 2009. No ano de 2006, Marlon viajou com Chris Pugh e Graham Charles (02-01-1950), renomado cactófilo inglês, de Stamford, especialista no gênero Gymnocalycium e editor do periódico The Cactus Explorers; em 2009, excursionou com Wiebe Bosma, da Holanda.

João Larocca e Marlon Machado descobriram Parodia gaucha nas serranias de Encruzilhada do Sul, que é, até o momento, a última espécie de cacto descrita para o Rio Grande do Sul (Machado et. al, 2008).

Igualmente notáveis são os trabalhos realizados por Anabela Silveira de Oliveira Deble (11-08-1969, Dom Pedrito) e Leonardo Paz Deble (09-06-1980, Bagé) no tocante aos cactos do Bioma Pampa. Em "O Bioma Pampa: contribuições científicas", de 2011, e "Bioma Pampa: ambiente e sociedade", de 2012, o casal de botânicos contribuiu com capítulos sobre a família Cactaceae no Rio Grande do Sul e um levantamento realizado em Lavras do Sul, com diversas espécies (Oliveira Deble, 2011; Jacinto et Oliveira Deble, 2012). Outro grupo interessado em cactos pampianos reúne Alberto Magli (02-05-1960, Bologna) e Giovanna Anceschi (05-06-1963, Milão), que percorreram diversos habitats no Rio Grande do Sul e Uruguai, entre os anos de 2006 e 2011, desenvolvendo estudos sobre conservação, distribuição geográfica e taxonomia (Magli, comunicação pessoal, 2016). Também deve ser mencionado, aqui, o trabalho investigativo de entusiastas, como Adriano Rudi Maixner (Porto Alegre),
Aoacy Antônio Orson e seu irmão Alcides Orson (Souza, Paraíba), o casal Ângela e Frederico Jaekel (Pelotas), Hari Konrad e seu filho Luiz Konrad (Imigrante), Helmut Horst Hessen (Crailsheim), Henrique Mallmann Büneker (Estrela), João Luiz De Nardin (Viamão), Jones Caldas da Silva (Porto Alegre), Sérgio Henrique Klein (Novo Hamburgo) e Wolfgang Prauser (Hanover).

No Uruguai, destacam-se as recentes contribuições dos botânicos Pablo Berazategui (0904-1972) e Williams Duarte de Barros (10-031953), dedicadas, sobretudo, a aspectos de fitogeografia e taxonomia (Berazategui, comunicação pessoal, 2016).

Por último, mas não menos importante, menciona-se a Fundação Zoobotânica do Rio Grande do Sul, instituição oficial de pesquisas do estado sulino, sediada em Porto Alegre, e que, desde 1997, através do "Projeto Pró-Guaíba", conta com uma nova casa de vegetação para abrigar sua coleção de cactos e outras plantas (Carneiro et. al, 2016). As expedições para coleta de material e as pesquisas realizadas neste período incrementaram, notavelmente, a coleção e o conhecimento da flora cactácea gaúcha. Em 1998, veio a lume o fascículo "Flora do Rio Grande do Sul - Cactos", levantando a importância do estudo e conservação das espécies nativas.

Atualmente, a Fundação Zoobotânica possui um acervo digital que ultrapassa $30 \mathrm{mil}$ fotografias de plantas em habitat natural e sob cultivo (Ramos, comunicação pessoal, 2016). Além disso, possui um acervo, em herbário, e uma impecável coleção viva ex-situ, organizada e mantida ao longo dos anos, especialmente por Ari Nilson e Paulo Gross, conhecida como "Módulo Xico Stockinger", em homenagem a Francisco Stockinger, que disponibilizou recursos para inúmeras viagens de pesquisa, em busca de cactos.

Em 30-03-2016 foi lançado o livro Cactos do Rio Grande do Sul, o qual, além de ricamente ilustrado e cheio de detalhes, é pioneiro neste setor da flora gaúcha. O trabalho, elaborado 
ao longo de quase quinze anos, foi financiado pelo Banco Mundial, através do "Projeto RS Biodiversidade", e desenvolvido por Ari Delmo Nilson, Ricardo Aranha Ramos (04-10-1961, Porto Alegre), Andreia Carneiro e Rosana Singer-Farias. Trata-se de um instrumento que há muito se fazia sentir, com vistas à popularização do conhecimento sobre as cactáceas do estado e, também, como veículo de sensibilização ambiental, face ao rico contingente endêmico, existente na paisagem sul-rio-grandense.

Por fim, cabe salientar que a investigação sobre os cactos do Rio Grande do Sul e Uruguai, apesar do extraordinário avanço demonstrado ao longo dos três artigos desta série, ainda está longe de se dar por concluída, restando muita pesquisa de campo a ser feita em busca de novas áreas de ocorrência e, até mesmo, de eventuais novos táxons, além de questões taxonômicas, fitogeográficas e filogenéticas. Lacunas, como estas, constituem vigoroso estímulo aos pesquisadores - e não apenas aos que atualmente labutam na seara -, mas, inclusive, à nova geração que haverá de surgir e dar seguimento à longa jornada em busca de um melhor conhecimento sobre estas plantas de agreste beleza e singulares formas, que tanto intrigam os cientistas e apaixonam homens e mulheres de todos os cantos do mundo.

Neste contexto - e como destaque a lamentar-se -, salienta-se a triste (e injusta) situação presentemente vivenciada na Fundação Zoobotânica do Rio Grande do Sul. É realmente desolador ver-se que essa instituição, de tantas e tão notáveis realizações em benefício da ciência e de vital importância para o futuro do Rio Grande do Sul, esteja sendo tratada levianamente pelos políticos de plantão, como se fosse um problema a ser extirpado e não como órgão indispensável ao futuro do estado. Nem mesmo os versos do poeta, ao advertir que "amanhã há de ser outro dia", serve de consolo ao ultraje cometido ao meio científico, pois não se recupera o tempo perdido e, muito menos, a dispersão e/ou extravio de um acervo penosamente reunido.

\section{AGRADECIMENTOS}

AAlberto Magli, Ari Delmo Nilson, Andreas Hofacker, Andreas Hamester, Eloísa Tregnagno, Jussara Stockinger, João Larroca, Konrad Herm, Kurt Ingo Horst, Marino Gustavo Hamester, Pablo Berazategui, Ricardo Aranha Ramos, Rudi Werner Büneker, Stanislav Stuchlík e Wolf-Rainer Abraham, bem como ao professor Marcelo Antônio Rodrigues, do Colégio Politécnico da Universidade Federal de Santa Maria, que viabilizou o necessário suporte financeiro para a realização desta pesquisa.

\section{REFERÊNCIAS}

ABRAHAM, W. R. Nieuwbeschrijving Notocactus scopa (Sprengel) Berger ex Backeberg var. xiphacanthus Abraham var. nov. Succulenta, v. 69, n. 12, p. 256-260, 1987.

ABRAHAM, W. R. Nieuwbeschrijving Notocactus scopa var. machadoensis Abraham var. nov. Succulenta, v. 67, n. 4, p. 81-84, 1988a.

ABRAHAM, W. R. Nieuwbeschrijving Notocactus scopa var. xicoi Abraham var. nov. ein nieuwe, Braziliaanse variëteit van Notocactus scopa. Succulenta, v 67, n. 5, p. 111-114, 1988b.

ABRAHAM, W. R. Nieuwbeschrijving Notocactus rudibuenekeri Abraham spec. nov. Succulenta, v. 67, n. 6 , p. 133-138, 1988c.

ABRAHAM, W. R. Wigginsia schaeferiana Abraham et. Theunissen spec. nov., eine neue flachbeibende Art aus Montevideo. Internoto, v.9, n. 1, p. 21-25, 1988d.

ABRAHAM, W. R. Erstbeschreibung Notocactus rubrigemmatus Abraham. Kakteen und andere Sukkulenten, v. 39, n. 2, p. 38-41, 1988e.

ABRAHAM, W. R. Notocactus gutierrezii, ein neuer rotblühhender Notocactus aus Brasilien. Kakteen und andere Sukkulenten, v. 39, n. 7, p. 150-154, 1988f.

ABRAHAM, W. R. Notocactus scopa var. marchesii, eine Stark sprossende Varietät von Notocactus scopa. Kakteen und andere Sukkulenten, v. 40, n. 7, p. 174-176, 1989.

ABRAHAM, W. R. Nieuwbeschrijving Frailea buenekeri spec. nov. Abraham. Succulenta, v. 69, p. 64-68, 1990a.

ABRAHAM, W. R. Nieuwbeschrijving Notocactus linkii var. albispinus var. nov. Abraham. Succulenta, v. 69, p. 117-120, 1990b. 
ABRAHAM, W. R; BÜNEKER, R. W. Erstbeschreibung Notocactus megapotamicus var. multicolorispinus. Kakteen und andere Sukkulenten, v. 43, n. 1, p. 20-22, 1992.

ALVES, J. F. Stockinger - vida e obra. Porto Alegre, 2012, 308 p.

ANDERSON, E. F. The cactus family. Timber Press, $2001.776 \mathrm{p}$.

BAUER, D; WAECHTER, J. L. Sinopse taxonômica de Cactaceae epifíticas no Rio Grande do Sul, Brasil. Acta botanica brasílica, v. 20, n. 1, p.225239, 2006.

BERGNER, S. Erweiterung des N. laetvirens um die Forma densispinus Bergner forma nova. Internoto, v. 14, n. 1, p. 3-12,1993.

BRAUN, P. Erstbeschreibung Notocactus graessneri fa. microdasys. Kakteen und andere Sukkulenten, v. 37, n. 3, p. 57. 1986.

BRAUN, P. J; ESTEVES PEREIRA, E. Kakteen und andere Sukkulenten in Brasilien. Schumannia, n. 3, 2001. 235 p.

CARNEIRO, A.M.; FARIAS-SINGER, R; RAMOS, R.A; NILSON, A.D. Cactos do Rio Grande do Sul. Porto Alegre: Fundação Zoobotânica do Rio Grande do Sul, 2016. 224 p.

DUARTE, B. A natureza transpira dos quadros de Gutierrez. Zero Hora. Segundo Caderno. Porto Alegre, 27 de dezembro, 1993.

FUNDAÇÃO ZOOBOTÂNICA DO RIO GRANDE DO SUL. Histórico do Jardim Botânico de Porto Alegre. Guia do visitante Jardim Botânico de Porto Alegre, p. 12-17, 2005.

FUNDAÇÃO ZOOBOTÂNICA DO RIO GRANDE DO SUL. Diagnóstico. Plano diretor do Jardim Botânico de Porto Alegre, p. 19-32, 2014.

GERLOFF, N. Wir stellen vor: Francisco Stockinger - Künstler + Kakteenfreund. Internoto, v. 9, n. 3, p. 80-81, 1988.

GERLOFF, N. Notocactus scopa (Sprengel) Berger ex Backeberg var. cobrensis Gerloff var. nova - Ein Kurzdornig, gruppenbildende Varietät des Notocactus scopa. Internoto, v. 11, n. 1, p. 3-9, 1990a.

GERLOFF, N. Notocactus incomptus Gerloff spec. nova. Internoto, v. 11, n. 2-3, p. 37-41, 1990 b.

GERLOFF, N. Notocactus glomeratus Gerloff spec. nova. Internoto, v. 12, n. 1, p. 3-10, 1991.

GERLOFF, N. In Memorian Heinz Werner RuoffVetsch. Internoto, v. 15, n. 2, p. 72, 1994a.
GERLOFF, N. Notocactus langsdorffii fa. eddiewarasii Gerloff. Minimus, v. 25, n. 2, p. 512, 1994b.

GERLOFF, N. Notocactus neohorstii (F. Ritter) Theun. var. prestlei N. Gerloff var. nova. Internoto, v. 16, n. 1, p. 3-17, 1995.

GERLOFF, N. Notocactus arachnitis v. minor Ritter forma darilhoensis fa. nova Gerloff. Internoto, v. 18, n. 4, p. 119-127, 1997.

GERLOFF, N. Vorgestellt: Csaba Kadar. Internoto, v. 31, n. 3, p. 86-87, 2010.

GERLOFF, N; KÖNINGS, G. Notocactus uebelmannianus Buining var. pleiocephalus Gerloff et Könings var. nova - Eine vielköpfige Varietät des Notocactus uebelmannianus aus Rio Grande do Sul (Brasilien). Internoto, v. 13, n. 4 , p. 103-112, 1992.

GERLOFF, N; NEDUCHAL, J. STUCHLÍK, S. Notokakteen - Gesamtdarstellung aller Notokakteen. 1995. 208 p.

GERLOFF, N.; NEDUCHAL, J. Taxonomische Neubearbeitung der Gattung Notocactus Friè. Internoto, v. 25, 2004. 128 p.

GERLOFF, N; NILSON, A. D. Notocactus calvescens N. Gerloff et A. D. Nilson spec. nova. Internoto, v. 14, n. 5, p. 75-83, 1994.

HERM, K. Notocactus herteri Werdermann forma pseudoherteri (Buining) Herm comb. nov. Internoto, v. 10, n. 2, p. 35-39, 1989.

HERM, K. Am Standort von Notocactus concinnus (Monville) Berger ex Backberg var. rubrigemmatus (Abraham) Gerloff. Internoto, v.11. 1, p. 28-23, 1990.

HERM, K. Die Pflanzengruppe um Notocactus horstii Internoto, v. 11, n.2-3, , p. 64-67, 1990.

HERM, K. Notocactus horstii und Verwandte. Internoto, v. 14, n. 2, p. 59-64 1993.

HERM, K. Ein Leben für die Kakteen - Zum Tode von Werner Uebelmann. Internoto, v. 35, n. 2, p. 47-48, 2014.

HERM, K; HOFACKER, A. CHARLES, G; VAN HEEK, W; BOHLE, B; STECKER, W; HEIMEN, G. Kakteen in Brasilien. Hügelsheim, 2000. $176 \mathrm{p}$.

HOFACKER, A. Ein neuer Standort von $N$. arechavaletai (Speg.) var. horstii Ritter. Internoto, v. 11, n. 4, p. 124-128, 1990.

HOFACKER, A. Notokakteen - von Acanthocephala bis Wigginsia. Deutsche-Kakteen Gesellschaft, 2013. 144 p. 
HOFACKER, A; BRAUN, P. J. Nomenclatural adjustments in Parodia. In: Cactaceae Consensus Initiatives, n. 6, 1998.

HOFACKER, A; HERM, K. Notocactus minusculus spec. nov. Internoto, v. 17, n. 3, p. 75-79, 1996.

HUNT, D. The New Cactus Lexicon. Milborne Port. 2006.

JACINTO, D. G; OLIVEIRA DEBLE, A. S. Levantamento das cactáceas no Cerro do Graxaim, Dom Pedrito RS. In: OLIVEIRA DEBLE, A. S, DEBLE, L. P; LEÃO, A.L.S (Orgs.) O Bioma Pampa: ambiente x sociedade. Bagé: Ediurcamp, 2012. p. 160-171.

$\mathrm{KOCH}, \mathrm{C}$. E. Erstbeschreibung Notocactus vilanovensis spec. nov. Internoto, v. 24, n. 2, p. 13-20, 2003

KÖNINGS, G. Notocactus uebelmannianus Buining var. nilsonii Könings var. nov. - Eine neue Varietät aus Rio Grande do Sul/ Brasilien. Internoto, v. 11, n. 4, p. 119-124, 1990.

LARROCCA, J. Cactáceas em paredões rochosos da Serra Geral do Rio Grande do Sul: uma abordagem fitogeográfica. Dissertação de Mestrado. UFRGS, Porto Alegre, 1998.

LISAL, K; KOLARIK, J. Erstbeschreibung $N$. arnostianus Lisal et Kolarik spec. nov. Internoto, v. 7, n.1, p. 4-5, 1986.

LISAL, K; KOLARIK, J. Erstbeschreibung $N$. ritterianus Lisal et Kolarik spec. nov. Internoto, v. 7, n. 1, p. 6-19, 1986.

MACHADO, M.C; NYFFELER, R; EGGLI, U; LARROCA, J.F. A new species of Parodia (Cactaceae, Notocacteae) from Rio Grande do Sul, Brasil. Novon, a Journal for Botanical Nomenclature, v. 18, n. 2, p. 214-129, 2008.

MACHADO, M. C. Fascinating Frailea, Part 1: General impressions. Cactus World - Britsh Cactus \& Succulent Society, v. 25, n. 1, 2007a.

MACHADO, M. C. Fascinating Frailea, Part 2: Review of the species from Rio Grande do Sul.
Cactus World - Britsh Cactus \& Succulent Society, v. 25, n. 2, 2007 b.

OLIVEIRA DEBLE, A. S. A família Cactaceae Juss. No Bioma Pampa - Checklist, distribuição geográfica e status de conservação. In: DEBLE, L; OLIVEIRA DEBLE, A. S; LEÃO, A.L.S (Orgs.). O Bioma Pampa - contribuições científicas. Bagé: Ediurcamp, 2011. p. 43-71.

PRAUSER, W. Abschied von Karl Heinz Prestlé. Internoto, v. 37, n. 2, p. 8, 2016.

PRESTLÉ, K. H. Auf Kakteensuche in Rio Grande do Sul (1). Internoto, v.1, n. 1-2, p. 7-10, 1980.

PRESTLÉ, K. H. Auf Kakteensuche in Rio Grande do Sul (2). Internoto, v. 2, n 1, p. 13-16. 1981.

PRESTLÉ, K. H. Auf Kakteensuche in Rio Grande do Sul (3). Internoto, v.2, n.2, p. 25, 1981.

PRESTLÉ, K. H. Erstbeschreibung Notocactus ibicuiensis Preslté spec. nov. Internoto, v.6, n. 4, p. 99-103, 1985a.

PRESTLÉ, K. H. Nieuwbeschrijving Notocactus stockingeri species nova. Suculenta, v. 64, n. 11, p. 226-229, 1985 b.

PRESTLÉ, K. H. Erstbeschreibung Notocactus meonacanthus Prestlé. Internoto, n.2, p. 35-39, 1986a.

PRESTLÉ, K. H. Erstbeschreibung Notocactus macambarensis Prestlé. Internoto, n.3, p. 67-72, 1986b.

PRESTLÉ, K. H. Nieuwbeschrijving Notocactus gibberulus spec. nov. Succulenta, v. 65, n. 6-7, p. 142-145, 1986c.

PRESTLÉ, K. H. Die Gymnocalycien des brasiliansichen Südens, einschliesslich der Vorkommen in Uruguay. 1991, 23 p.

RUOFF, H. Notocactus ottonis fa. tranqueras HR 88. Internoto, v. 10, n. 3, , p. 89-91, 1989.

STUCHLÍK, S. Rod Notocactus - Monographie. 1993. 176p.

STUCHLÍK, S. Kakteen Rio Grande do Sul. Kaktusy, special, n. 1, 2010. 35p. 